Research, Hebrew SeniorLife \& Harvard Medical School, Boston, MA, USA, 8. Institute for Aging Research, Hebrwe SeniorLife \& BIDMC, Harvard Medical School, Boston, MA, USA

Analytic decisions can profoundly impact observational study results. Little data exist about the influence of these decisions when estimating effects of osteoporosis medications in older adults. We evaluated the effects of first-line osteoporosis treatment, bisphosphonates, versus calcitonin on hip fractures and esophagitis among NH residents using several approaches to address biases. Approaches included propensity score matching, inverse probability of treatment weighting (IPW), multiple imputation (MI), machine learning, and Fine and Gray competing risk regressions. Results did not vary markedly by method for hip fractures, but did for esophagitis. Hazard ratios for hip fracture ranged from 0.86 [95\% CI, 0.71-0.91] for MI plus IPW to 0.89 (CI, 0.75-1.05) for Fine and Gray models. Esophagitis estimates ranged from 0.95 (CI, 0.78-1.17) for MI plus IPW to 1.19 (CI, 0.90-1.58) for Fine and Gray models. Investigators studying medication effects in observational studies should use several complementary analytic methods and should present all results.

\section{BALANCING THE BENEFITS OF BISPHOSPHONATE TREATMENT WITH RISK OF ADVERSE EVENTS IN FRAIL NURSING HOME RESIDENTS}

K. McConeghy ${ }^{1}$, Y. Lee, MS $\mathrm{MPH}^{2}$, A.R. Zullo, $\mathrm{PhD}^{3}$, T. Zhang, $\mathrm{PhD}^{4}$, S.D. Berry, MD MPH${ }^{5}$, 1. Brown University School of Public Health, Providence, Rhode Island, United States, 2. Brown University School of Public Health, Providence, RI, USA, 3. Brown University School of Public Health, Providence, RI, USA, 4. Brown University School of Public Health, Providence, RI, USA, 5. Institute for Aging Research, Hebrew SeniorLife \& BIDMC, Harvard Medical School, Boston, MA, USA

$\mathrm{NH}$ residents are at significant risk of adverse drug reactions (ADRs) due to frailty, decreased drug clearance and drug-drug interactions. Oral bisphosphonates (BP) cause mild-moderate GI irritation which may lead to esophagitis. Additionally, we hypothesize GI irritation may increase risk of C. difficile infection (CDI). We evaluated the risk of hospitalization for one conventional (esophagitis, ICD9 530.1) and one novel (CDI, ICD9 008.45) ADR in an intent-totreat approach of elderly NH residents newly prescribed a bisphosphonate versus calcitonin, 1:1 matched with propensity scores. Among 5,209 bisphosphonate users, the hazard ratios (HR) were 1.11 (95\% Confidence Interval [CI], 0.84, 1.47) for esophagitis and 1.15 (95\% CI: 0.82, 1.61) for CDI. Bisphosphonate use was not associated with significant risk of esophagitis or CDI. Future efforts will focus on other potential ADRs (e.g. atypical femur fractures) in order to better appreciate the net clinical benefit of these drugs in the $\mathrm{NH}$ population.

\section{TRENDS IN THE INCIDENCE OF HIP FRACTURES AND POST-FRACTURE MORTALITY AMONG U.S. NURSING HOME RESIDENTS, 2007 TO 2013}

L. Daiello ${ }^{1}$, Y. Lee, MS MPH ${ }^{2}$, D.P. Kiel, MD $\mathrm{MPH}^{3}$, S.D. Berry, $\mathrm{MD} \mathrm{MPH}^{4}$, 1. Brown University, School of Public Health, Providence, Rhode
Island, United States, 2. Brown Universtiy School of Public Health, Providence, RI, USA, 3. Brown University School of Public Health, Providence, RI, USA, 4. Institute for Aging Research, Hebrew SeniroLife \& BIDMC, Harvard Medical School, Boston, MA, USA

The incidence of hip fractures in community-dwelling older adult populations declined substantially between 2002 and 2012; however, little is known about longitudinal changes in hip fracture rates and post-fracture mortality among nursing home $(\mathrm{NH})$ residents. This study investigated trends in fracture rates and mortality among 1.9 million $\mathrm{NH}$ residents from 2007 to 2013 . We found that the crude incidence rate of hip fractures decreased from 3.55/100 person-years in 2007 to $3.17 / 100$ person-years in 2013. The age-adjusted hip fracture and one-year mortality rates were $14 \%$ [incidence rate ratio, $1.14,95 \%$ confidence interval (CI), 1.10-1.17] and $7 \%$ [hazard ratio, $1.07,95 \%$ CI, 1.02-1.12] greater in 2007 than in 2013, respectively. To our knowledge, this is the first national study describing declining trends in hip fractures and mortality among NH residents. These results are encouraging, but additional research is needed to identify the most effective strategies to reduce hip fractures and improve longterm outcomes.

\section{INCIDENCE OF HIP FRACTURE IN NATIVE} AMERICAN RESIDENTS OF U.S. NURSING HOMES T. Zhang ${ }^{1}$, O. Amir, MD MPH, BIDMC 2 , A.R. Zullo, PhD ${ }^{3}$, D.P. Kiel, $\mathrm{MD} \mathrm{MPH}^{4}$, S.D. Berry, $\mathrm{MD} \mathrm{MPH}^{5}$, 1. Brown University School of Public Health, Providence, Rhode Island, United States, 2. Harvard Medical School, Boston, MA, USA, 3. Brown University School of Public Health, Providence, RI, USA, 4. Institute for Aging Research, Hebrwe SeniorLife \& BIDMC, Harvard Medical School, Boston, MA, USA, 5. Institute for Aging Research, Hebrew SeniorLife \& BIDMC, Harvard Medical School, Boston, MA, USA

The U.S NH population is increasingly more ethnically diverse. Racial disparities in health care outcomes exist in this setting. Our study compared the incidence rate (IR) of hip fracture in U.S. Native American (NA) NH residents with incidence in other ethnicities, including blacks and whites. Ethnic-specific IRs for hip fracture were standardized using inverse probability weights, estimated using $>60$ resident and 6 facility level characteristics. Among 1,136,262 NH residents, 4,391 (0.4\%) were NA, 980,559 (86.3\%) were white, and 116,098 (10.2\%) were black. Adjusted IRs of hip fractures were $2.16 / 100$ person-years (95\% CI 1.92-2.45) among NA residents compared to 2.05 (95\% CI 2.03-2.07) in whites and $0.82(95 \%$ CI $0.79-0.85)$ in blacks. Over $50 \%$ of NAs vs. $31.2 \%$ of whites resided in a rural facility. NA NH residents had the highest rates of hip fracture. Facility characteristics (e.g., rural location) may contribute to observed racial disparity and merit further investigation.

\section{SESSION 705 (SYMPOSIUM)}

\section{MECHANISMS UNDERLYING PHYSICAL FRAILTY IN HUMANS}

Chair: D. Melzer, University of Exeter Medical School \& University of Connecticut Health Center, Exeter, England 